\title{
Structural Optimization Using the Grouping Method and the 1/3rd Rule Based on Specific Strain Energy
}

\author{
Young-Doo Kwon ${ }^{* 1}$, Jin-Won Lee ${ }^{\# 2}$, Jin-Sik Han ${ }^{\# 3}$ \\ ${ }^{1}$ School of Mechanical Engineering \& IEDT, Kyungpook National University, Daegu, 41566, Korea \\ ${ }^{2}$ Powertrain Installation, Volvo Group Korea Co., Ltd., Changwon, 51710, Korea \\ ${ }^{3}$ Department of Mechanical Engineering, The Graduate School of Kyungpook National University, Daegu, \\ 41566, Korea \\ 1ydkwon@knu.ac.kr \\ ${ }^{2}$ mouse2000won@naver.com \\ ${ }^{3}$ hjs121987@gmail.com \\ ${ }^{*}$ School of Mechanical Engineering \& IEDT, Kyungpook National University, Daegu, 41566, Korea \\ *ydkwon@knu.ac.kr
}

\begin{abstract}
Structural optimization approaches may be categorized into three major types. One type of approach is topological optimization, which involves many sensitivity analysis variables. This type of approach sometimes results in odd shapes, such as checkerboard patterns. The other types are shape optimization and parametric optimization, which involve certain difficulties in dealing with the selection of proper parameters and require repeated meshing for the purpose of finite element analysis. We propose an efficient method for grouping finite elements to reduce the number of degrees of freedom of the system considerably and to perform the optimization of several groups of elements. If we reject elements using a cutoff criterion based on the specific strain energy for several steps, we may obtain a topologically optimized result for the discrete configuration, without any irregularity. This optimization may have a higher-speed process based on the grouping method. The grouping method divides the elements into three groups on the basis of strain energy - a high-energy group, a low-energy group, and a mid-energy group. By moving the high-energy group to a high-priority group and eliminating the lowenergy group, a 1/3rd rule can be used to obtain an optimized design. The 1/3rd rule is fast and effective and provides a way to obtain a realistic result. Several examples were considered to test the optimization efficacy of the grouping technique.
\end{abstract}

Keyword- Grouping Method, 1/3rd rule, Strain Energy, Structural Optimization

\section{INTRODUCTION}

The importance of optimization [1], which can be used to remove unnecessary parts and improve the effectiveness of a design, has grown gradually in engineering design. Structural optimization approaches may be categorized into three major types. One type of approach is topological optimization, which considers many variables in a sensitivity analysis [2]-[4]. This type of approach sometimes results in odd shapes, such as checkerboard patterns. The other two major types of approaches are shape optimization and parametric optimization. These types of approaches have certain difficulties in dealing with the selection of proper parameters and require repeated meshing for finite element analysis. This is a complex and time-consuming execution method, and a single execution involves considerable effort and cost. Therefore, producing objects using this approach involves considerable costs and time expenditures, which decreases its economic efficiency.

Therefore, it is necessary to develop a new and more realistic optimization method. In this paper, we propose an efficient method for grouping finite elements to reduce the number of degrees of freedom of the system and performing optimization for several groups of elements. If we reject elements using a cutoff criterion based on the specific strain energy [5]-[9] for several steps, we can obtain a topologically optimized result with a discrete configuration and without any irregularity. This method exhibits fast calculation times and yields realistic results.

In addition, a $1 / 3$ rd rule based on strain energy was developed. The $1 / 3$ rd rule divides elements into groups and compares them in terms of their strain energy, retaining the high-energy groups and removing the lowenergy groups. The $1 / 3$ rd rule increases the understanding of the structure by examining it in terms of the strain energy, and thereby increases the efficiency of the structure. In addition, the results obtained are practical because the operator considers the entire shape at each step in the process. 


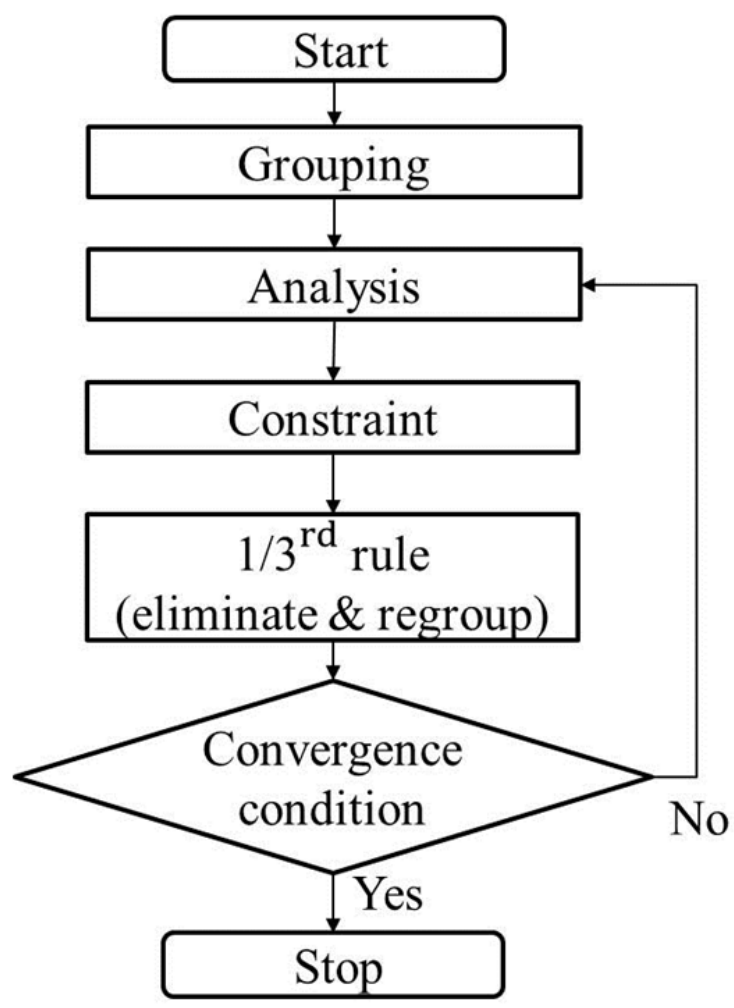

Fig. 1. Flowchart of optimization procedure

Several examples were considered to test the efficiency of the grouping technique and application of the $1 / 3 \mathrm{rd}$ rule to optimization. A cantilever structure was fully optimized using PIAnO [10]. Then, its elements were grouped into several categories and optimized with far less computational effort. We then applied several rejection steps to obtain topologically optimized results.

\section{GRouping MethoD AND $1 / 3^{\text {RD }}$ RULE}

In topological optimization, the number of variables is high, and the computation cost is high as a result. Satisfying the strength constraint is another problem that needs to be addressed, as well as addressing the checkerboard pattern problem. In this section, a grouping method is proposed for use in resolving these types of problems to a certain extent. The procedure for applying the grouping method and $1 / 3$ rd rule is as follows:

1 The entire domain of a model is classified into several groups.

1-1Each group is selected manually based on similar levels of strain energy density, based on experience and mechanics.

1-2Every group is discretized into many finite elements.

2 The finite element model is analyzed after the application of boundary conditions. The strength constraint is satisfied by adjusting element thicknesses.

3 The elements in each group are sorted in order of their strain energy density (SED).

4 The groups are sorted in order of the highest SED of the elements within each group or in the order of the highest average SED.

5 The highest SEDs of each group are compared with the highest SED of the 1st sorted group, and the groups of the highest SED smaller than 1/3rd of the highest SED are eliminated from the model.

6 Elements in the groups with the highest SED smaller than 2/3 of the highest SED are modified, depending upon the level in the group.

6-1The elements with SEDs in the top 1/3rd are moved to the adjacent group.

6-2The elements with SEDS in the middle 1/3rd remain in the same group.

6-3The elements in the lowest $1 / 3$ rd are removed from the group.

7 The process returns to step 2 and repeats until there is no group whose highest SED is smaller than $1 / 3$ rd of the first highest SED. 


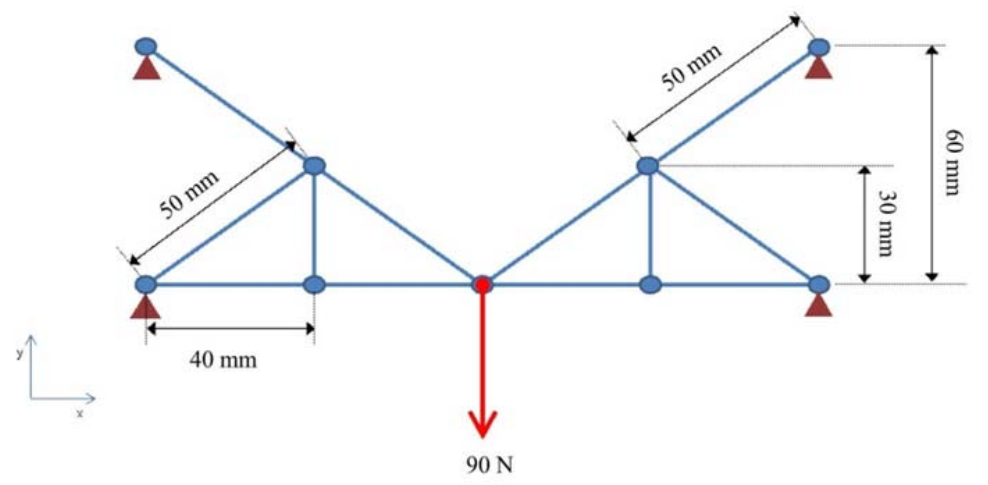

Fig. 2. Truss structure model

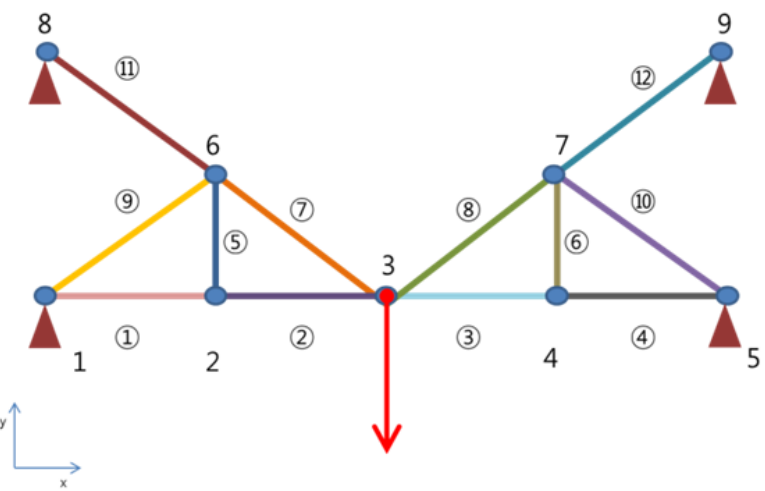

Fig. 3. Numbering of elements and nodes

Through the actions of elimination and modification (elements being, moved removed, or unchanged), the overall model is topologically optimized while the strength constraint is satisfied. The chance of a checkerboard pattern occurring and the necessity for smoothing during post-processing are minimized by the reasonable selection of groups for the model. A flowchart of the procedure is shown in Fig. 1.

\section{EXAPMPLES}

Three examples - truss, cantilever, and clamped deep beam problems - are presented to demonstrate the effectiveness of the grouping method.

\section{A. Truss}

The first example is a truss structure, illustrated in Fig. 2 and Fig. 3. If the results obtained and time required for the calculations for each element are compared with the results obtained using the grouping method, the efficiency of the grouping method is easily understood. The truss structure consists of steel with a Young's modulus of $200 \mathrm{GPa}$ and a Poisson's ratio of 0.3 . As mentioned above, based on engineering judgment and experience, the elements under stress in a similar direction are grouped together. If the elements are not grouped, the number of elements is 12 . However, as shown in Table 1, if the elements acting in similar directions are grouped together based on symmetry, they are categorized into six groups. In addition, among the six grouped elements, those acting in a similar direction are regrouped. This results in a total of four elements, so four elements are generated. The amount of calculation required is thus reduced to one third of the original amount required. This example is a simple case. Because the amount of calculation required is reduced by grouping the elements, the calculation time is decreased, and similar results are obtained.

TABLE I

Numbering of Non-grouped and Grouped Elements

\begin{tabular}{|c|c|c|c|c|c|c|c|c|c|c|c|c|}
\hline nodes & 1,2 & 2,3 & 3,4 & 4,5 & 2,6 & 4,7 & 3,6 & 3,7 & 1,6 & 5,7 & 6,8 & 7,9 \\
\hline $\begin{array}{c}12 \\
\text { elements }\end{array}$ & (1) & (2) & (3) & (4) & 5 & 6 & 7 & 8 & 9 & (10 & (11) & (12) \\
\hline 6 groups & $\mathbf{1}$ & $\mathbf{2}$ & $\mathbf{2}$ & $\mathbf{1}$ & $\mathbf{4}$ & $\mathbf{4}$ & $\mathbf{5}$ & $\mathbf{5}$ & $\mathbf{3}$ & $\mathbf{3}$ & $\mathbf{6}$ & $\mathbf{6}$ \\
\hline 4 groups & $\mathrm{I}$ & $\mathrm{I}$ & $\mathrm{I}$ & $\mathrm{I}$ & $\mathrm{III}$ & $\mathrm{III}$ & $\mathrm{IV}$ & $\mathrm{IV}$ & $\mathrm{II}$ & $\mathrm{II}$ & IV & IV \\
\hline
\end{tabular}


TABLE III

Results of the Calculations with Non-grouped Elements

\begin{tabular}{|c|c|c|c|}
\hline Element No. & $\mathrm{A}\left(\mathrm{mm}^{2}\right)$ & $\mathrm{L}(\mathrm{mm})$ & volume $\left(\mathrm{mm}^{3}\right)$ \\
\hline 1 & $8.23 \mathrm{E}-04$ & 40 & 0.0329 \\
\hline 2 & $7.38 \mathrm{E}-04$ & 40 & 0.0295 \\
\hline 3 & $1.01 \mathrm{E}-03$ & 40 & 0.0403 \\
\hline 4 & $2.03 \mathrm{E}-04$ & 30 & 0.0061 \\
\hline 5 & $7.79 \mathrm{E}-04$ & 30 & 0.0234 \\
\hline 6 & $4.29 \mathrm{E}-05$ & 50 & 0.0021 \\
\hline 7 & $1.50 \mathrm{E}+01$ & 50 & 750.0000 \\
\hline 8 & $1.50 \mathrm{E}+01$ & 50 & 750.0000 \\
\hline 9 & $6.53 \mathrm{E}-04$ & 50 & 0.0326 \\
\hline 10 & $9.37 \mathrm{E}-03$ & 50 & 0.4687 \\
\hline 11 & $1.50 \mathrm{E}+01$ & 50 & 750.0000 \\
\hline 12 & $1.50 \mathrm{E}+01$ & 50 & 750.0000 \\
\hline
\end{tabular}

TABLE IIIII

Results of the Calculation with Six-group Elements

\begin{tabular}{|c|c|c|c|c|c|c|}
\hline Group No. & G1 & G2 & G3 & G4 & G5 & G6 \\
\hline A $\left(\mathrm{mm}^{2}\right)$ & $1.24 \mathrm{E}-09$ & $2.58 \mathrm{E}-06$ & $2.12 \mathrm{E}-09$ & $1.55 \mathrm{E}-09$ & $1.50 \mathrm{E}+01$ & $1.50 \mathrm{E}+01$ \\
\hline $\mathrm{L}(\mathrm{mm})$ & 40 & 40 & 50 & 30 & 50 & 50 \\
\hline Volume $\left(\mathrm{mm}^{3}\right)$ & $4.97 \mathrm{E}-08$ & $1.03 \mathrm{E}-04$ & $1.06 \mathrm{E}-07$ & $4.64 \mathrm{E}-08$ & $7.50 \mathrm{E}+02$ & $7.50 \mathrm{E}+02$ \\
\hline
\end{tabular}

TABLE IVV

Results of the Calculation with Four-group Elements

\begin{tabular}{|c|c|c|c|c|}
\hline Group No. & G1 & G2 & G3 & G4 \\
\hline A $\left(\mathrm{mm}^{2}\right)$ & $1.34 \mathrm{E}-09$ & $1.00 \mathrm{E}-09$ & $1.00 \mathrm{E}-09$ & $1.50 \mathrm{E}+01$ \\
\hline $\mathrm{L}(\mathrm{mm})$ & 40 & 50 & 30 & 50 \\
\hline Volume $\left(\mathrm{mm}^{3}\right)$ & $5.35 \mathrm{E}-08$ & $5.00 \mathrm{E}-08$ & $3.00 \mathrm{E}-08$ & $7.50 \mathrm{E}+02$ \\
\hline
\end{tabular}

As Tables 2 to 4 show, the results of the calculations are almost the same, but the number of elements required for the calculation is noticeably reduced. Based on this, the calculation time is also reduced. The effect of the grouping method is greater if the target object is more complicated and the number of elements is increased. The results of the truss problem demonstrate the efficiency of the grouping method.

\section{B. Cantilever}

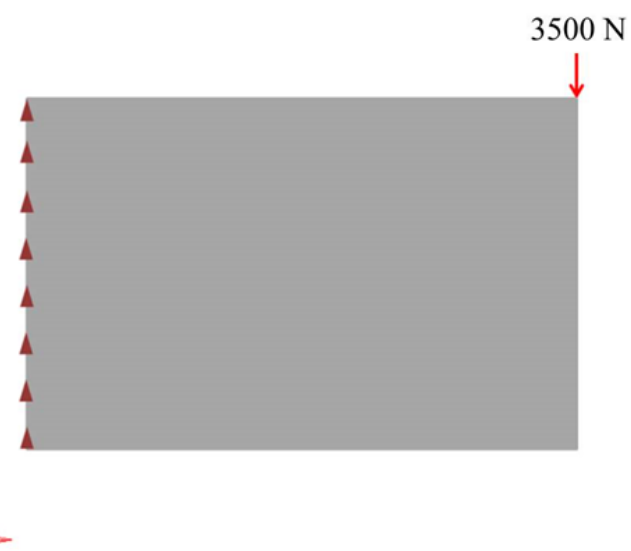

Fig. 4. Boundary conditions and load on cantilever 


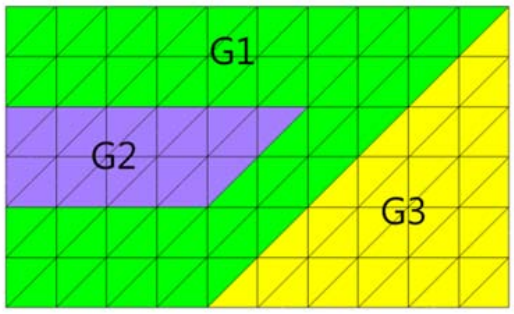

Fig. 5. Grouped elements

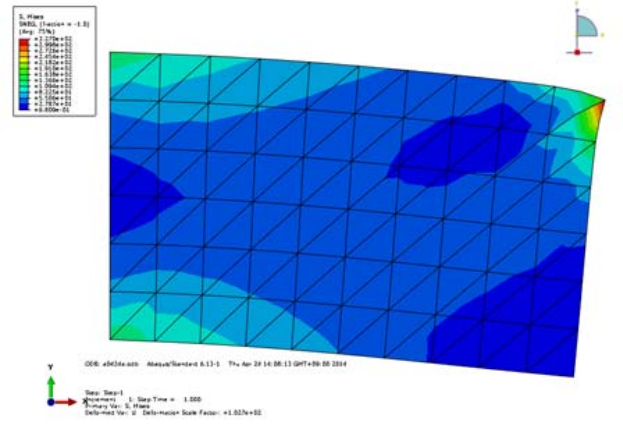

(a) von Mises stress

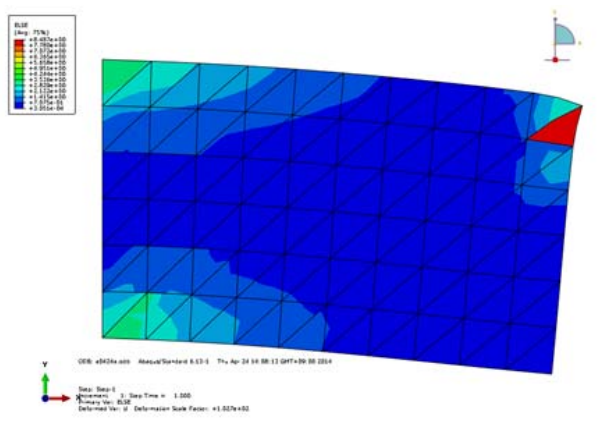

(b) Strain energy

Fig. 6. Analysis results

Figure 4 illustrates a cantilever problem that can be solved using the grouping method and the 1/3rd rule. This cantilever has the following properties. The type of steel is AISI 8000 series steel, with a density of 7.8e-9 $\mathrm{Mg} / \mathrm{mm} 3$ and a Young's modulus of $207 \mathrm{GPa}$. Its Poisson's ratio is 0.3 , and its yield strength is $900 \mathrm{MPa}$. This cantilever is under a load of 3,500 N, as shown Fig. 4. The objective of the problem is to minimize the weight of the cantilever while satisfying the requirement for a safety factor of three. This example is used to demonstrate that the grouping method and the 1/3rd rule may be the best method for optimizing the design of this type of structure.

The cantilever problem is solved by applying the grouping method to a large bundle of elements rather than individual elements. The elements of the cantilever problem are grouped empirically as shown in Fig. 5. For this shape, the von Mises stress and the strain energy are obtained using Abaqus, as shown in Fig. 6.

By creating a table for the strain energy per unit area (SED) and per element, as shown in Table 5, the sizes and priorities of the element groups can be determined. In considering the entire shape, high-strain-energy elements move to the high level, and low-strain-energy elements are eliminated. Mid-level elements are maintained without any decision being made concerning their placement.

TABLE V

Strain Energy Sheet for the First-step Model

\begin{tabular}{|c|c|c|c|c|c|}
\hline \multicolumn{2}{|c|}{ Group 1 } & \multicolumn{2}{c|}{ Group 2 } & \multicolumn{2}{c|}{ Group 3 } \\
\hline Element & SED & Element & SED & Element & SED \\
\hline 6 & 0.2780 & 73 & 0.0277 & 120 & 0.6789 \\
\hline 63 & 0.2720 & 69 & 0.0275 & 118 & 0.1544 \\
\hline 14 & 0.1985 & 76 & 0.0270 & 119 & 0.1046 \\
\hline 62 & 0.1854 & 77 & 0.0261 & 85 & 0.0733 \\
\hline 64 & 0.1845 & 72 & 0.0258 & 117 & 0.0519 \\
\hline 7 & 0.1829 & 80 & 0.0245 & 86 & 0.0455 \\
\hline 5 & 0.1773 & 78 & 0.0220 & 87 & 0.0453 \\
\hline
\end{tabular}




\begin{tabular}{|c|c|c|c|c|c|}
\hline 8 & 0.1597 & 81 & 0.0219 & 116 & 0.0431 \\
\hline 22 & 0.1532 & 74 & 0.0219 & 107 & 0.0352 \\
\hline 13 & 0.1440 & 75 & 0.0213 & 109 & 0.0320 \\
\hline 15 & 0.1412 & 83 & 0.0212 & 88 & 0.0318 \\
\hline & \multirow{4}{*}{. } & 65 & 0.0207 & \multirow{4}{*}{$\begin{array}{l}\cdot \\
\cdot \\
\cdot \\
\cdot\end{array}$} & \multirow{4}{*}{$\begin{array}{l}\cdot \\
\cdot \\
.\end{array}$} \\
\hline . & & 79 & 0.0205 & & \\
\hline . & & 71 & 0.0197 & & \\
\hline & & 82 & 0.0193 & & \\
\hline 41 & 0.0225 & 70 & 0.0184 & 115 & 0.0205 \\
\hline 57 & 0.0211 & 84 & 0.0181 & 96 & 0.0170 \\
\hline 43 & 0.0200 & 68 & 0.0173 & 104 & 0.0150 \\
\hline 48 & 0.0199 & 67 & 0.0127 & 95 & 0.0140 \\
\hline 54 & 0.0186 & 66 & 0.0095 & 114 & 0.0110 \\
\hline 42 & 0.0186 & & & 103 & 0.0083 \\
\hline 51 & 0.0180 & & & 94 & 0.0082 \\
\hline 49 & 0.0163 & & & 113 & 0.0058 \\
\hline 60 & 0.0149 & & & 102 & 0.0050 \\
\hline 50 & 0.0127 & & & 112 & 0.0015 \\
\hline 55 & 0.0103 & & & 101 & 0.0014 \\
\hline 59 & 0.0093 & & & 111 & 0.0007 \\
\hline 56 & 0.0089 & & & 110 & 0.0000 \\
\hline
\end{tabular}

According to Table 5, the strain energies of the elements in group 2 are generally low, as are the von Mises stresses. Therefore, group 2 does not seem to be important to the overall shape and is eliminated. The highstrain-energy elements in group 3 move to the high group, and the low-strain-energy elements in group 3 are eliminated. The results of the above procedures are shown in Fig. 7. The optimal thickness that satisfies the constraint is found using PIAnO.

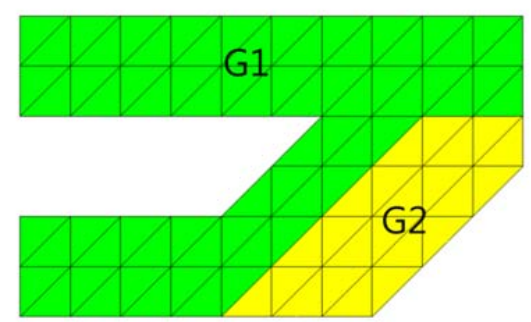

Fig. 7. Results of applying the $1 / 3$ rd rule to the first step of the cantilever problem 


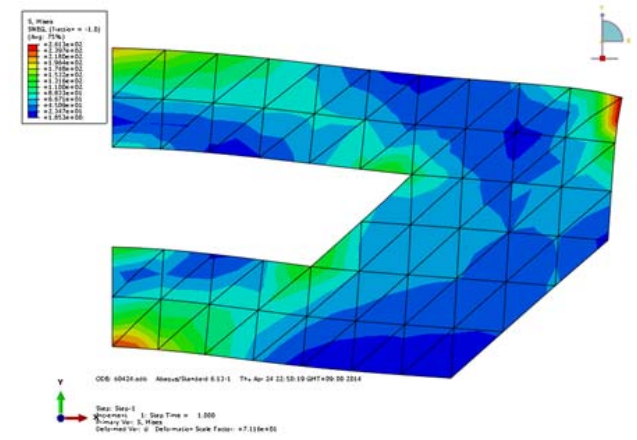

(a) von Mises stress

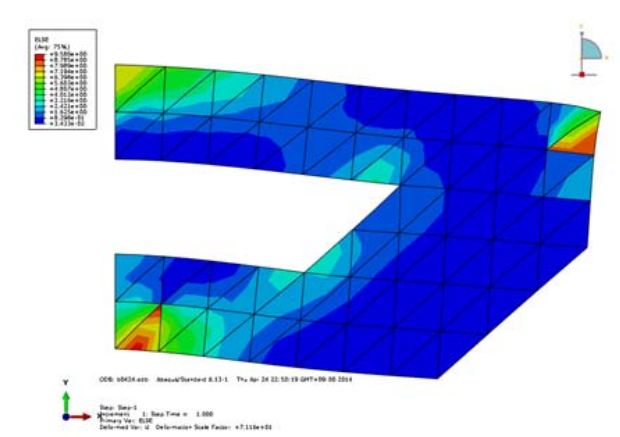

(b) Strain energy

Fig. 8. Analysis results for regrouped cantilever

Application of the $1 / 3$ rd rule to the first step results in division into two groups, as shown in Fig. 7. For this shape, the von Mises stress and the strain energy obtained using Abaqus are shown in Fig. 8.

After the von Mises stresses and SED values for the above results were determined, the SED sheet shown in Table 6 was developed, and the 1/3rd rule was applied.

TABLE VI

Strain Energy Sheet for the Second-step Model

\begin{tabular}{|c|c|c|c|}
\hline \multicolumn{2}{|c|}{ Group 1} & \multicolumn{2}{|c|}{ Group 2} \\
\hline Element & SED & Element & SED \\
\hline 12 & 22.6965 & 29 & 2.2643 \\
\hline 121 & 22.2012 & 17 & 2.2445 \\
\hline 24 & 16.2038 & 32 & 2.2064 \\
\hline 120 & 15.1352 & 41 & 2.1345 \\
\hline 122 & 15.0598 & 20 & 2.1075 \\
\hline 13 & 14.9302 & 44 & 1.9978 \\
\hline 11 & 14.4742 & 42 & 1.7998 \\
\hline 14 & 13.0344 & 54 & 1.7874 \\
\hline 36 & 12.5100 & 30 & 1.7854 \\
\hline 23 & 11.7536 & 31 & 1.7394 \\
\hline 25 & 11.5272 & 56 & 1.7316 \\
\hline . & . & 5 & 1.6921 \\
\hline . & . & 43 & 1.6724 \\
\hline . & . & 19 & 1.6101 \\
\hline 78 & 1.9857 & 55 & 1.5737 \\
\hline 66 & 1.8373 & 18 & 1.5043 \\
\hline 95 & 1.7238 & 68 & 1.4802 \\
\hline 69 & 1.6339 & 8 & 1.4163 \\
\hline 79 & 1.6241 & 7 & 1.0366 \\
\hline 92 & 1.5222 & 6 & 0.7728 \\
\hline 67 & 1.5191 & & \\
\hline 82 & 1.4682 & & \\
\hline 80 & 1.3306 & & \\
\hline
\end{tabular}




\begin{tabular}{|c|c|l|l|}
\hline 107 & 1.2186 & & \\
\hline 81 & 1.0349 & & \\
\hline 93 & 0.8436 & & \\
\hline 106 & 0.7626 & & \\
\hline 94 & 0.7274 & & \\
\hline
\end{tabular}

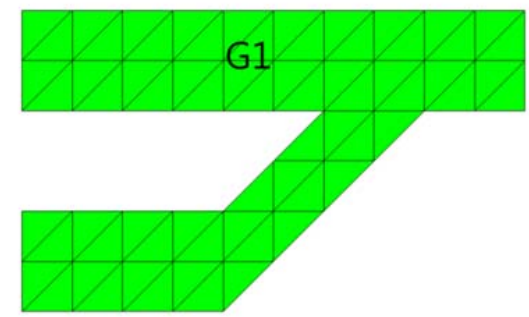

Fig. 9. Final shape of the cantilever model

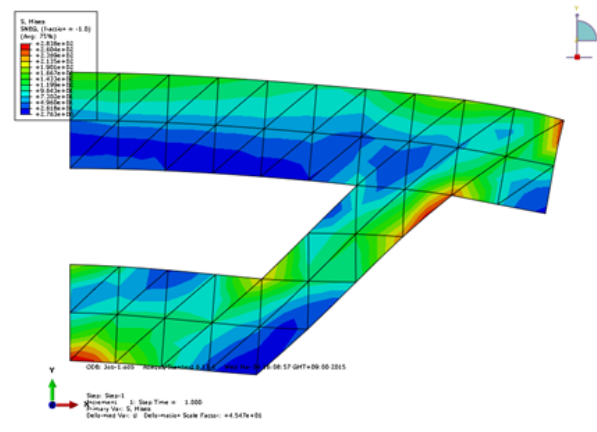

(a) von Mises stress

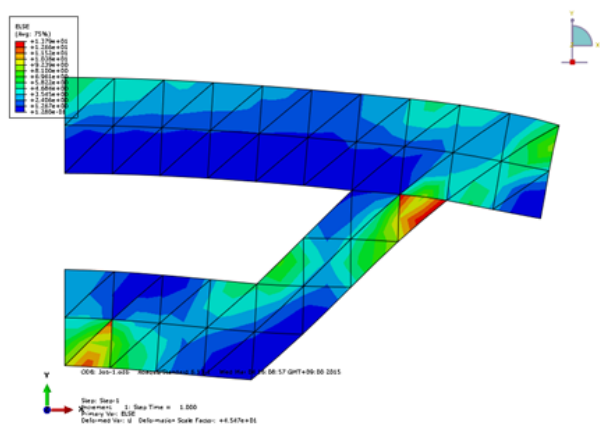

(b) Strain energy

Fig. 10. Analysis results for final shape of cantilever

According to the $1 / 3$ rd rule, group 2 is eliminated. The optimized result that satisfies the constraints is identified using PIAnO. The final shape is shown in Fig. 9. The weight of this structure is $40 \%$ less than that before the optimization.

The above shape was analyzed again using Abaqus and optimized once again using PIAnO. The following results were obtained.

In the results shown in Fig. 10, the strain energy is distributed evenly, and the weight has been reduced overall. This shape was optimized once again to calculate the optimal thickness and weight. The results are shown in Table 7.

This result shows that the weight has decreased by approximately $41.39 \%$ satisfying the constraint of maximum von Mises stress $300 \mathrm{MPa}$. Through this cantilever problem, the efficiency of grouping can be reconfirmed.

TABLE VII

Thickness Results Obtained from Optimization

\begin{tabular}{|c|c|c|c|}
\hline & $\sigma_{\max }(\mathrm{MPa})$ & weight $(\mathrm{mg})$ & thickness $(\mathrm{mm})$ \\
\hline Step 1 & 300 & $1.275 \mathrm{e}-04$ & 10.8988 \\
\hline Step 2 & 300 & $9.964 \mathrm{e}-05$ & 10.8723 \\
\hline Step 3 & 300 & $7.473 \mathrm{e}-05$ & 11.2724 \\
\hline
\end{tabular}




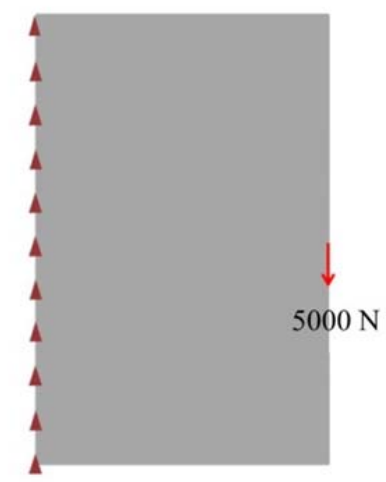

Fig. 11. Boundary condition and load on clamped deep beam

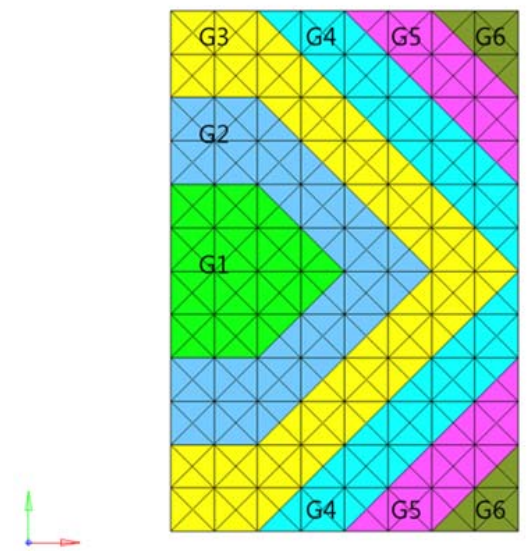

Fig. 12. Grouped elements of clamped deep beam

\section{Clamped deep beam}

The next example is the clamped deep beam problem illustrated in Fig. 11. Its vertical length is greater than that of the cantilever, and the location of the load is at the center of the structure. The size of the clamped deep beam is $40 \mathrm{~mm} \times 60 \mathrm{~mm}$, and the left side is fixed. The clamped deep beam is subjected to a vertical downward $5000-\mathrm{N}$ load at the center of the right side. As with the cantilever beam, the objective is to find a shape with an optimum weight while satisfying the constraint of not exceeding the allowable stress of $300 \mathrm{MPa}$.

The elements for the shape shown in Fig. 11 were assembled using Hypermesh, and the elements were divided into groups empirically, as shown in Fig. 12. Because the load is located at the center of the clamped deep beam, the shapes of the groups are symmetric upward and downward.

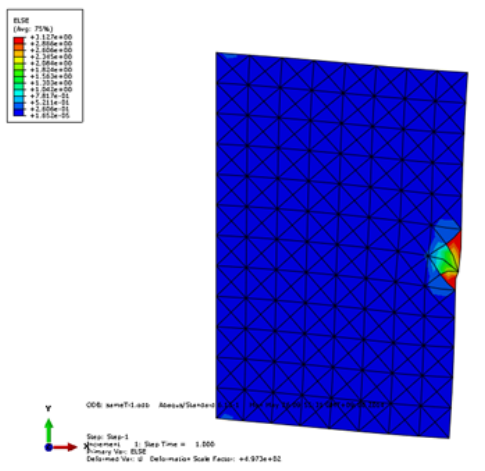

(a) von Mises stress

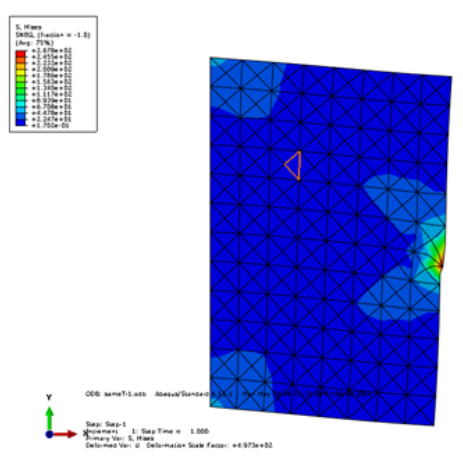

(b) Strain energy

Fig. 13. Analysis results 
The von Mises stresses and strain energies for the grouped elements were obtained using Abaqus, as shown in Fig. 13.

Based on the listing of elements and SEDs shown in Table 8, elements were eliminated or moved according to the $1 / 3$ rd rule. The results obtained are shown in Fig. 14.

TABLE VIII

Strain Energy Sheet of First-step Model

\begin{tabular}{|c|c|c|c|c|c|c|c|c|c|c|c|}
\hline \multicolumn{2}{|c|}{ Group 1} & \multicolumn{2}{|c|}{ Group 2} & \multicolumn{2}{|c|}{ Group 3} & \multicolumn{2}{|c|}{ Group 4} & \multicolumn{2}{|c|}{ Group 5} & \multicolumn{2}{|c|}{ Group 6} \\
\hline Element & SED & Element & SED & Element & SED & Element & SED & Element & SED & Element & SED \\
\hline 113 & 0.007 & 58 & 0.011 & 360 & 0.188 & 723 & 0.500 & 731 & 0.006 & 740 & 0.0001 \\
\hline 485 & 0.007 & 448 & 0.011 & 722 & 0.188 & 359 & 0.500 & 351 & 0.006 & 342 & 0.0001 \\
\hline 66 & 0.007 & 10 & 0.011 & 2 & 0.046 & 724 & 0.084 & 732 & 0.005 & 695 & 0.0001 \\
\hline 440 & 0.007 & 400 & 0.011 & 408 & 0.046 & 358 & 0.084 & 350 & 0.005 & 291 & 0.0001 \\
\hline 67 & 0.007 & 59 & 0.010 & 1 & 0.034 & 726 & 0.050 & 687 & 0.004 & 739 & 0.0001 \\
\hline 439 & 0.007 & 447 & 0.010 & 405 & 0.034 & 356 & 0.050 & 299 & 0.004 & 343 & 0.0001 \\
\hline 116 & 0.007 & 105 & 0.010 & 357 & 0.030 & 727 & 0.034 & 688 & 0.003 & 696 & 0.0000 \\
\hline 486 & 0.007 & 493 & 0.010 & 721 & 0.030 & 355 & 0.034 & 298 & 0.003 & 290 & 0.0000 \\
\hline 118 & 0.006 & 57 & 0.010 & 3 & 0.027 & 725 & 0.029 & 734 & 0.003 & 742 & 0.0000 \\
\hline 484 & 0.006 & 445 & 0.010 & 407 & 0.027 & 353 & 0.029 & 348 & 0.003 & 340 & 0.0000 \\
\hline . & . & . & . & . & . & . & . & . & . & 741 & 0.0000 \\
\hline$\cdot$ & . & · & · & · & · & $\cdot$ & · & $\cdot$ & · & 337 & 0.0000 \\
\hline . & . & . & . & . & . & . & . & . & . & 743 & 0.0000 \\
\hline 529 & 0.006 & 16 & 0.006 & 201 & 0.009 & 551 & 0.005 & 692 & 0.001 & 339 & 0.0000 \\
\hline 433 & 0.005 & 394 & 0.006 & 589 & 0.009 & 147 & 0.005 & 294 & 0.001 & 744 & 0.0000 \\
\hline 20 & 0.005 & 167 & 0.006 & 149 & 0.009 & 595 & 0.005 & 647 & 0.001 & 338 & 0.0000 \\
\hline 390 & 0.005 & 531 & 0.006 & 545 & 0.009 & 199 & 0.005 & 243 & 0.001 & & \\
\hline 23 & 0.005 & 213 & 0.006 & 152 & 0.008 & 596 & 0.005 & 648 & 0.001 & & \\
\hline 387 & 0.005 & 577 & 0.006 & 546 & 0.008 & 198 & 0.005 & 242 & 0.001 & & \\
\hline 17 & 0.005 & 215 & 0.005 & 262 & 0.007 & 642 & 0.005 & 737 & 0.001 & & \\
\hline 389 & 0.005 & 579 & 0.005 & 628 & 0.007 & 248 & 0.005 & 341 & 0.001 & & \\
\hline 22 & 0.005 & 261 & 0.005 & 309 & 0.006 & 641 & 0.004 & 738 & 0.001 & & \\
\hline 388 & 0.005 & 625 & 0.005 & 673 & 0.006 & 245 & 0.004 & 344 & 0.001 & & \\
\hline 24 & 0.004 & 216 & 0.005 & 263 & 0.005 & 597 & 0.004 & 694 & 0.000 & & \\
\hline 386 & 0.004 & 578 & 0.005 & 627 & 0.005 & 193 & 0.004 & 292 & 0.000 & & \\
\hline 21 & 0.004 & 264 & 0.004 & 312 & 0.004 & 598 & 0.004 & 693 & 0.000 & & \\
\hline 385 & 0.004 & 626 & 0.004 & 674 & 0.004 & 196 & 0.004 & 289 & 0.000 & & \\
\hline
\end{tabular}




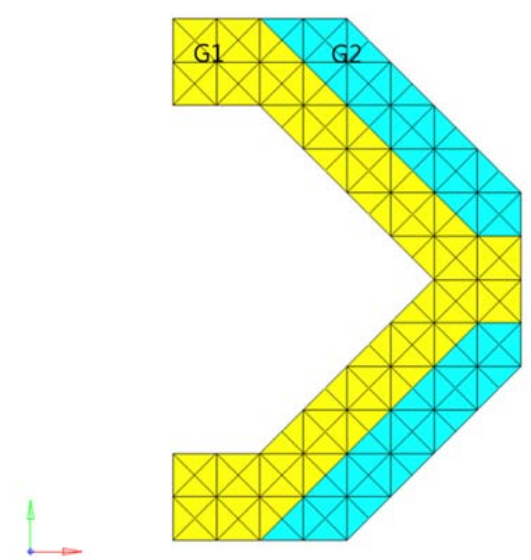

Fig. 14. The results of the $1 / 3$ rd rule, first step, for the clamped deep beam

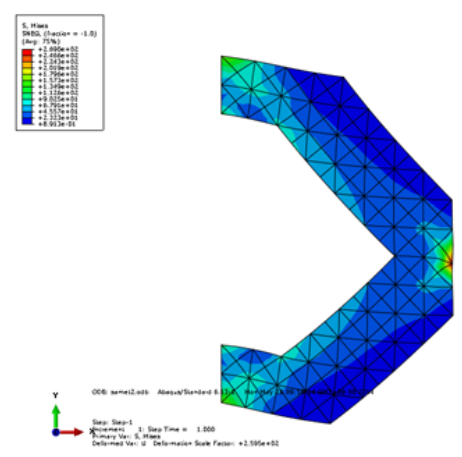

(a) von Mises stress

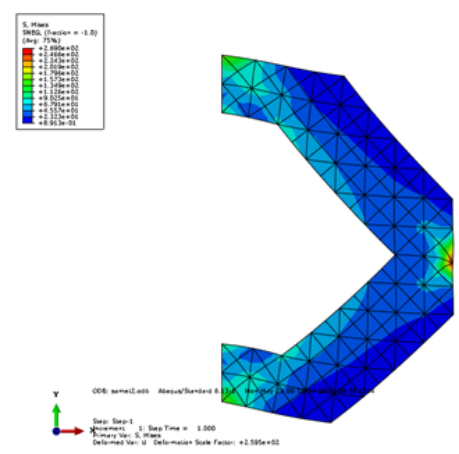

(b) Strain energy

Fig. 15. Analysis results for regrouped clamped deep beam

These results were analyzed again using Abaqus, as shown in Fig. 15. Because considerable portions of the structure still appeared to be unnecessary, we decided to apply the $1 / 3$ rd rule one more time.

After listing the SEDs and elements, as shown in Table 9, low-strain elements were eliminated, and highstrain elements were moved to the high level, according to the $1 / 3 \mathrm{rd}$ rule.

TABLE IX

Strain Energy Sheet of Second-step Model

\begin{tabular}{|c|c|c|c|}
\hline \multicolumn{2}{|c|}{ Group 1 } & \multicolumn{2}{c|}{ Group 2 } \\
\hline Element & SED & Element & SED \\
\hline 359 & 9.58832 & 726 & 0.870392 \\
\hline 723 & 9.58832 & 725 & 0.616489 \\
\hline 2 & 7.211136 & 679 & 0.573113 \\
\hline 408 & 7.211136 & 680 & 0.425903 \\
\hline 1 & 4.261984 & 635 & 0.319316 \\
\hline 405 & 4.261984 & 636 & 0.30694 \\
\hline 360 & 4.239464 & 682 & 0.292934 \\
\hline 722 & 4.239464 & 591 & 0.279462 \\
\hline 3 & 3.173456 & 592 & 0.264418 \\
\hline 407 & 3.173456 & 727 & 0.253897 \\
\hline 106 & 2.637224 & 681 & 0.251197 \\
\hline
\end{tabular}




\begin{tabular}{|c|c|c|c|}
\hline$\cdot$ & $\cdot$ & $\cdot$ & $\cdot$ \\
$\cdot$ & $\cdot$ & $\cdot$ & $\cdot$ \\
$\cdot$ & $\cdot$ & $\cdot$ & $\cdot$ \\
\hline 204 & 0.559075 & 251 & 0.069972 \\
\hline 590 & 0.559075 & 148 & 0.065785 \\
\hline 312 & 0.552746 & 300 & 0.05884 \\
\hline 674 & 0.552746 & 250 & 0.051003 \\
\hline 305 & 0.545626 & 145 & 0.050018 \\
\hline 677 & 0.545626 & 297 & 0.038077 \\
\hline 152 & 0.532826 & 199 & 0.02597 \\
\hline 546 & 0.532826 & 198 & 0.014447 \\
\hline 253 & 0.520713 & 196 & 0.013047 \\
\hline 633 & 0.520713 & 245 & 0.012496 \\
\hline 256 & 0.516326 & 146 & 0.012038 \\
\hline 634 & 0.516326 & 248 & 0.011893 \\
\hline 149 & 0.486726 & 193 & 0.009099 \\
\hline 545 & 0.486726 & 147 & 0.00799 \\
\hline
\end{tabular}

The results obtained are shown in Fig. 16 (a). To illustrate the advantages of the grouping method and the $1 / 3$ rd rule, the results obtained using the conventional topology method for a clamped deep beam are shown Fig. 16 (b) [11].

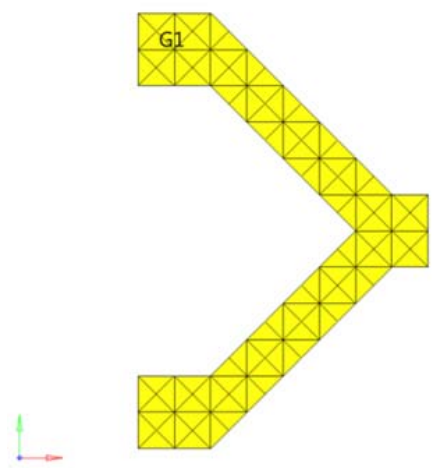

(a) Final shape obtained using the grouping method

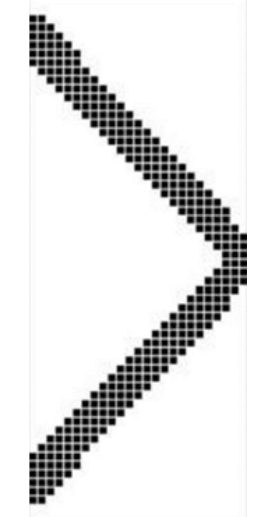

(b) Final shape obtained using a conventional method [11]

Fig. 16. Comparison of grouping method with $1 / 3$ rd rule and conventional topology method 


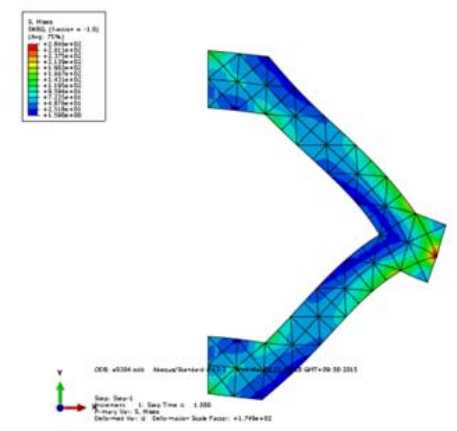

(a) von Mises stress

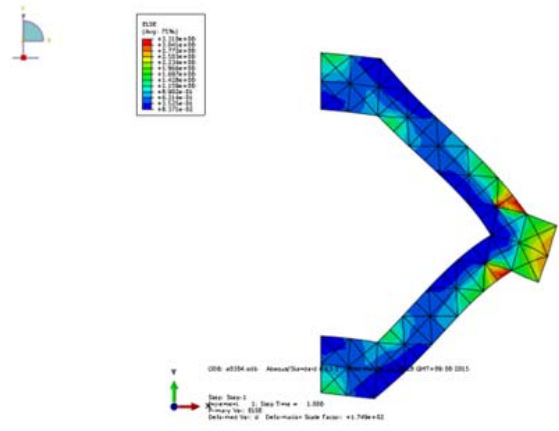

(b) Strain energy

Fig. 17. Analysis results for regrouped clamped deep beam

TABLE X

Thickness Optimization Result

\begin{tabular}{|c|c|c|c|}
\hline & $\sigma_{\max }(\mathrm{MPa})$ & weight $(\mathrm{Mg})$ & thickness $(\mathrm{mm})$ \\
\hline Step 1 & 300 & $2.012 \mathrm{e}-04$ & 10.7524 \\
\hline Step 2 & 300 & $1.008 \mathrm{e}-04$ & 10.7756 \\
\hline Step 3 & 300 & $6.212 \mathrm{e}-05$ & 10.9857 \\
\hline
\end{tabular}

The above results were analyzed again using Abaqus and optimized once again using PIAnO. The following results were obtained.

As Fig. 17 shows, the strain energy is evenly distributed, and the weight has been reduced overall. This shape was optimized once again to calculate the optimal thickness and weight. The results are shown in Table 10.

As the results show, the weight was decreased by $69.12 \%$ in comparison to the initial design of the beam.

\section{CONCLUSION}

In the grouping method developed in this study, engineers intervene directly in the optimization process so that the optimal solutions to realistic problems can be found. The general topology optimization approach satisfies the constraints and tunes itself to the objectives, but in fact, the shape obtained as a result often cannot be produced in reality. In grouping, engineers consider each element directly and can add one or leave one out; therefore, it is more efficient than any other optimization method. In addition, because the optimization is carried out based on the strain energy, the efficiency of the entire shape can be increased considerably. By eliminating unnecessary parts and moving some parts with high strain energy, the strain energy can be distributed evenly, so that an efficient design, without any unnecessary parts, is obtained by considering the contributions of all of the elements. The grouping method and the $1 / 3$ rd rule are more efficient than other optimization methods used in production because they make it possible for the engineer to identify and add or subtract each element directly.

The results of this study of the grouping optimization method can be summarized as follows.

1. The grouping method and 1/3rd rule examined in this study can quickly and accurately identify highly realistic and easily manufactured shapes by addressing the problems of the traditional topological optimization method, such as the complexity of the process and the impracticality of some of the results.

2. Analysis in terms of the strain energy per unit area increases the efficiency of the entire shape. By eliminating unnecessary parts and reinforcing high-strain-energy parts, the strain energy is evenly distributed, and the action of the entire shape is made structurally uniform. Thus, the structure can be designed efficiently without unnecessary parts.

3. In conventional topological optimization, a large number of finite element analyses are performed to identify and remove ineffective elements. The proposed grouping method requires several finite element analyses to obtain a reasonable shape in the structural, artistic, manufacturing, and practical senses.

The results obtained show that the grouping optimization method can be used to easily reduce the weight of the designed structure by more than $30 \%$, and avoid unrealistic structural designs and checkerboard patterns. Thus, the grouping optimization method makes it possible to identify the optimal structure in manufacturing practice. Moreover, because element identification is fast in the group optimization and because individual elements are identified in groups, the computational effort required is minimized. In conclusion, because engineers can intervene directly in each step, the grouping optimization method can be used to identify the optimal structural solution that satisfies a variety of conditions at the same time. 


\section{ACKNOWLEDGMENT}

This research was supported by the Basic Science Research Program of the National Research Foundation of Korea (NRF), funded by the Ministry of Education (NRF-2012R1A1A2008903). The authors were partially supported by the BK21 Plus project of the National Research Foundation of Korea.

\section{REFERENCES}

[1] J. S. Arora, Introduction to optimum design, 3rd ed., Boston: Academic Press, 2011.

[2] Y. D. Kwon, S. B. Jin, and J. Y. Kim, "Local zooming genetic algorithm and its application to radial gate support problems," Structural Engineering and Mechanics, vol. 17 (5), pp. 611-626, 2004.

[3] Y. D. Kwon, S. B. Kwon, H. M. Gil, and H. J. Cho, "The empirical corner stiffness for right-angle frames of rectangular and H-type cross-sections," Structural engineering and mechanics, vol. 51 (3), pp. 471-485, May. 2014.

[4] J. Lewin, Hydraulic gates and valves: in free surface flow and submerged outlets, 2nd ed., London: Thomas Telford, 2001.

[5] A. P. Boresi, R. J. Schmidt, Advanced mechanics of materials, 6th ed., New York: John Wiley \& Sons, 2003.

[6] J. M. Gere, S. P. Timoshenko, Mechanics of materials, 4th SI ed., Cheltenham: Stanley Thornes, 1999.

[7] H. Reismann, P. S. Pawlik, Elasticity: theory and applications, New York: John Wiley and Sons, 1980.

[8] S. P. Timoshenko, J. N. Goodier, Theory of Elasticity, 3rd ed., New York: McGraw-Hill, 1987.

[9] A. C. Ugural, S. K. Fenster, Advanced strength and applied elasticity, 4th ed., London: Prentice Hall, 2011.

[10] PIAnO User's Manual Version 3.3, PIDOTEC Inc., 2011

[11] S. Y. Wang, K. Tai, "Bar-system representation for topology optimization using genetic algorithms," Engineering Computations, vol. 22 (2), pp. 206-231, Mar. 2005.

\section{AUTHOR PROFILE}

Author1 Young-Doo Kwon Ph.D., Dept. of Mechanical Engineering \& IEDT, Kyungpook National University, KOREA.

Author2 Jin-Won Lee R\&D Engineer, Powertrain Installation, Volvo Group Korea Co., Ltd., Korea

Author3 Jin-Sik Han A graduate university student, Department of Mechanical Engineering, The Graduate School of Kyungpook National University, Korea 